\title{
Antifungal and antibacterial effects of newly created lactic acid bacteria associations depending on cultivation media and duration of cultivation
}

\author{
Lusine Matevosyan, Inga Bazukyan and Armen Trchounian * (D)
}

\begin{abstract}
Background: The newly created associations of lactic acid bacteria $(L A B)$ isolated from Armenian dairy products (yoghurt, sour cream and different varieties of cheese), as well as from the gastrointestinal tract of honeybees were screened according to their antifungal and antibacterial activity.

Results: $L A B$ strains were mixed at equal proportions (1:1) according to mathematical planning of experiments. Antifungal and antibacterial effects of different combinations (associations) were determined in different media, employing well-diffusion and total diffusion into agar methods. A number of fungal and bacterial test-organisms, including pathogenic ones, were used. Pure LAB strain cultures were used as a control. The antifungal effect of the most active strain Lactobacillus rhamnosus MDC 9661 in the associations with other LAB strains was partly decreased. At the same time, some mixed LAB cultures in DeMan, Rogosa and Sharpe (MRS) medium demonstrated significant antibacterial activity against wide spectra of test-organisms only in the case of simultaneous cultivation of $L A B$ strains. On the other hand, in the case of different $L A B$ strains cultivated in MRS with 24-h time break between mix formations by different strains, no inhibitory activity was revealed. But the inhibitory effect of many $L A B$ associations against test-organisms was significantly increased in the case of separated cultivation in milk.

Conclusion: The inhibitory effect of mixed LAB associations showed stronger dependence on the cultivation media and on the duration of cultivation with respect to each other. The co-cultivation of some strains, like L. rhamnosus MDC 9661, could lead to changed antagonistic activity. Consequently, the results are significant for creation and further investigation of $L A B$ associations, as effective probiotics, and for their probable application in the production of antimicrobial preparations.
\end{abstract}

Keywords: Lactic acid bacteria, Antifungal activity, Antibacterial activity, LAB associations

\section{Background}

Various pathogenic microorganisms, including fungi, which contaminate the nutrients of humans and animals, can be potentially dangerous agents and cause the loss of money [1]. The reducing contamination by various pathogenic bacteria, molds and yeasts in the production and storage of food and feed is of paramount importance, and the most interesting task is to develop the efficient

\footnotetext{
* Correspondence: trchounian@ysu.am

Department of Biochemistry, Microbiology and Biotechnology, Faculty of Biology, Yerevan State University, 1 Alex Manoogian Str, 0025 Yerevan, Armenia
}

(c) The Author(s). 2019 Open Access This article is distributed under the terms of the Creative Commons Attribution 4.0 International License (http://creativecommons.org/licenses/by/4.0/), which permits unrestricted use, distribution, and reproduction in any medium, provided you give appropriate credit to the original author(s) and the source, provide a link to the Creative Commons license, and indicate if changes were made. The Creative Commons Public Domain Dedication waiver (http://creativecommons.org/publicdomain/zero/1.0/) applies to the data made available in this article, unless otherwise stated. strates of both natural origin and well- investigated manufacturers are a key rule for choosing new agents. Lactic acid bacteria (LAB) best meet these requirements. In recent years, special interest in the antagonistic activity of bacterial communities is increased.

LAB have been used in the domestic food production for centuries not only because of they were tasty, but also because they allowed food to be stored for a long period of time. A lot of representatives of this group showed high antagonistic activity. The group of LAB is composed of the representatives of such genera, as 
Lactobacillus, Lactococcus, Streptococcus, Leuconostoc, Pediococcus, Enterococcus, Oenococcus, Weissella and others [2]. Of great interest is the genus Lactobacillus because of multiple antagonistic activities of its representatives. The list of antimicrobial metabolites synthesized by LAB includes organic acids, hydrogen peroxide, various lipids and bacteriocins [3]. LAB as the best probiotics were granted the status of Generally Regarded As Safe (GRAS) by the Food and Drug Administration (FDA) [4], and their benefits for human and animal gastrointestinal tract and immune system have been well known. However, the lack of the toxic effects of their metabolites is still under investigation $[5,6]$.

The crucially different from each other ecological-geographic conditions of Armenia lead to the development of unique communities of $\mathrm{LAB}$ in national food products. Several data about the antimicrobial effects of mixed LAB cultures against food-borne pathogens have been published [7-11]. However, their dependence on different factors, including media composition and duration of cultivation, was not shown clearly. It is very important to create correct associations, in which all strains will show the synergistic effect not only in simultaneous growth but also during the synthesis of active components. Various scenarios are possible in these associations: competition for nutrients, inhibition of one strain by other antibacterial components, inhibition of necessary actions due to the use of bacterial components instead of preliminary nutrients, etc. Unfortunately, less data on the pathway of metabolism in microbial associations is known. So this is the first problem to be solved. The study of mixed LAB cultures would explain the various microbial-microbial interactions that can be used in the production of food and drugs.

The aim of this work was the creation of various LAB associations from the previously studied strains which have strong antimicrobial effects separately. The dependence of antifungal and antibacterial effects of the LAB combinations on the composition of nutrient media and cultivation has been studied.

\section{Results}

\section{Antifungal activity of $L A B$ associations}

The results of the antifungal effects of individual $L A B$ strains were presented in previous studies [12], and were also increased in Table 1. A study of the antifungal activity of 15 different LAB associations showed that they have almost the same inhibitory effects on various types of mold and yeast (Table 2). Interestingly, inhibitory effect of $L$. rhamnosus MDC 9661 in combination with other LAB strains (VKPM B-3809, RIN-2003-Ls, INRA-2010-4.2, INRA-2010-5.2 and INR-2010-Tsov-G-St) was partly decreased (see Table 2, Mixes 1-5). Particularly, L. rhamnosus MDC 9661 has partly lost its activity against $P$. aurantioviolaceum and G. candidum in the mixture with S. thermophilus VKPM B-3809, as well as against $P$. aurantioviolaceum and A. flavus in the mixture with RIN-2003-Ls. The associations of MDC 9661 with INRA-2010-4.2 and INRA2010-5.2 couldn't inhibit the growth of $P$. aurantioviolaceum, T. viride and A. flavus. Finally, the mixture of MDC 9661 with INR-2010-Tsov-G-St did not show any activity against $A$. flavus. None of the associations can inhibit the growth of yeast. And only the combination of $L$. rhamnosus MDC 9661 with S. thermophilus VKPM B-3809 could inhibit the growth of A. flavus. Thus, we can conclude that the creation of associations with different strains of $\mathrm{LAB}$ and L. rhamnosus MDC 9661 is impractical; because in all associations, inhibition of the antifungal activity of MDC 9661 was observed.

\section{Antibacterial activity of $L A B$ associations}

The results of the antibacterial activity of individual LAB strains are presented in Table 3. Mixed cultures showed a significant difference depending on the type of cultivation and the type of growth medium. Interestingly, some $\mathrm{LAB}$ associations showed significant antibacterial activity in MRS against E. coli VKPM-M17, B. mesentericus WT, potentially pathogenic S. typhimurium MDC 1759, P. aeruginosa WT272786 and S. aureus MDC 5233 in the case of simultaneous cultivation of LAB strains (Fig. 1).

No inhibitory activity was observed against potential pathogenic strains in the case of time-spaced LAB cultivation in MRS. In this case, antibacterial effect was determined only against $M$. luteus WT and B. mesentericus WT (Fig. 2). Thus, the simultaneous cultivation of LAB strains can stimulate the production of antibacterial substances, enlarging their antibacterial activity.

Table 1 Antifungal activity of $L A B$ isolates

\begin{tabular}{|c|c|c|c|c|c|c|c|c|}
\hline Fungi LAB strain & M. plumbeus & P. aurantio-violaceum & Penicillumspp. & F. oxysporum & C. herbarum & T. viride & G. candidum & A. flavus \\
\hline MDC 9661 & + & + & + & + & + & + & + & $+/-$ \\
\hline RIN-2003-Ls & - & - & - & + & + & - & - & - \\
\hline MDC 9632 & - & - & - & + & + & - & - & - \\
\hline MDC 9633 & - & - & - & $+1-$ & - & - & - & - \\
\hline VKPM B-3809 & - & - & - & + & - & - & - & - \\
\hline INR-2010-Tsov-G-St & - & - & - & + & - & - & - & - \\
\hline
\end{tabular}

$(+)$ - presence of antifungal activity which means the inhibition of $10^{4}$ fungi spores, (-) - absence of antifungal activity, (+/-) - elongation of spore generation 
Table 2 Antifungal activity of $L A B$ associations

\begin{tabular}{|c|c|c|c|c|c|c|c|c|}
\hline $\begin{array}{l}\text { Fungi } \\
\text { Mix (1:1) }\end{array}$ & M. plumbeus & P. aurantio-violaceum & Penicillumspp. & F. oxysporum & C. herbarum & T. viride & G. candidum & A. flavus \\
\hline 1 & + & - & + & + & + & + & - & + \\
\hline 2 & + & - & + & + & + & + & + & - \\
\hline 3 & + & - & $+/-$ & + & + & - & + & - \\
\hline 4 & - & - & + & + & + & - & + & - \\
\hline 5 & + & + & + & + & + & + & + & - \\
\hline 6 & - & - & - & + & + & - & - & - \\
\hline 7 & - & - & - & - & - & - & - & - \\
\hline 8 & - & - & - & - & - & - & - & - \\
\hline 9 & - & - & - & + & $+/-$ & - & - & - \\
\hline 10 & - & - & - & + & - & - & - & - \\
\hline 11 & - & - & - & - & $+/-$ & - & - & - \\
\hline 12 & - & - & - & + & - & - & - & - \\
\hline 13 & - & - & - & - & - & - & - & - \\
\hline 14 & - & - & - & - & $+/-$ & - & - & - \\
\hline 15 & - & - & - & $+/-$ & - & - & - & - \\
\hline
\end{tabular}

$(+)$ - presence of antifungal activity which means the inhibition of $10^{4}$ fungi spores, $(-)$ - absence of antifungal activity, (+/-) - elongation of spore generation

Compared with cultivation in MRS, the inhibitory effect of many LAB associations was significantly increased in milk against E. coli VKPM-M17, S. aureus MDC 5233, B. mesentericus WT in the case of time-spaced strain cultivation (Fig. 3). The same composition of media and cultivation type affected the antibacterial activity of mixed LAB cultures. Interestingly, some mixed cultures in milk showed an inhibitory effect even against $B$. subtilis WT-A1 strain, but no activity was detected against potential pathogenic test-organisms. The simultaneous cultivation of LAB was not investigated in milk, because the strain MDC 9661 could not grow in milk within $24 \mathrm{~h}$.

\section{Discussion}

The study of antimicrobial activity of LAB strains, especially their mixed cultures, becomes more and more promising. Unfortunately, there are not so many published works about antimicrobial effects of mixed $L A B$ cultures; only a few data were published by different groups of researchers. Particularly, LAB associations

Table 3 Antibacterial activity of $\angle A B$ isolates

\begin{tabular}{|c|c|c|c|c|c|c|c|c|}
\hline $\begin{array}{l}\text { Test-organism } \\
\text { LAB strain }\end{array}$ & Medium & E. coli M17 & Salmonella typhimurium 1759 & M. luteus & P. aeruginosa & S. aureus & B. mesentericus & B. subtilis \\
\hline \multirow[t]{2}{*}{ MDC 9661} & Skim milk & $13^{*}$ & 16 & 18 & 13 & 17 & - & - \\
\hline & MRS & 11 & 14 & 10 & 10 & 18 & 10 & - \\
\hline \multirow[t]{2}{*}{ RIN-2003-LS } & Skim milk & 12 & 15 & 18 & 10 & 18 & - & 11 \\
\hline & MRS & 12 & 12 & 9 & 9 & - & 11 & - \\
\hline \multirow[t]{2}{*}{ MDC 9632} & Skim milk & 12 & 13 & 17 & - & 15 & 11 & 11 \\
\hline & MRS & 12 & 12 & 9 & 11 & - & 11 & - \\
\hline \multirow[t]{2}{*}{ MDC 9633} & Skim milk & 12 & 15 & 18 & 10 & 16 & 10 & - \\
\hline & MRS & 11 & 13 & 12 & 10 & 11 & 10 & - \\
\hline \multirow[t]{2}{*}{ VKPM B-3809 } & Skim milk & 10 & 12 & 16 & - & 12 & - & - \\
\hline & MRS & 12 & 12 & 9 & 10 & 18 & 10 & - \\
\hline \multirow[t]{2}{*}{ E.d } & Skim milk & 10 & 12 & 17 & - & 12 & - & - \\
\hline & MRS & 12 & 11 & 9 & 10 & 20 & 10 & - \\
\hline \multirow[t]{2}{*}{ B7 } & Skim milk & - & 12 & 17 & 9 & 15 & 9 & - \\
\hline & MRS & 11 & 15 & 9 & 11 & 13 & 16 & - \\
\hline
\end{tabular}




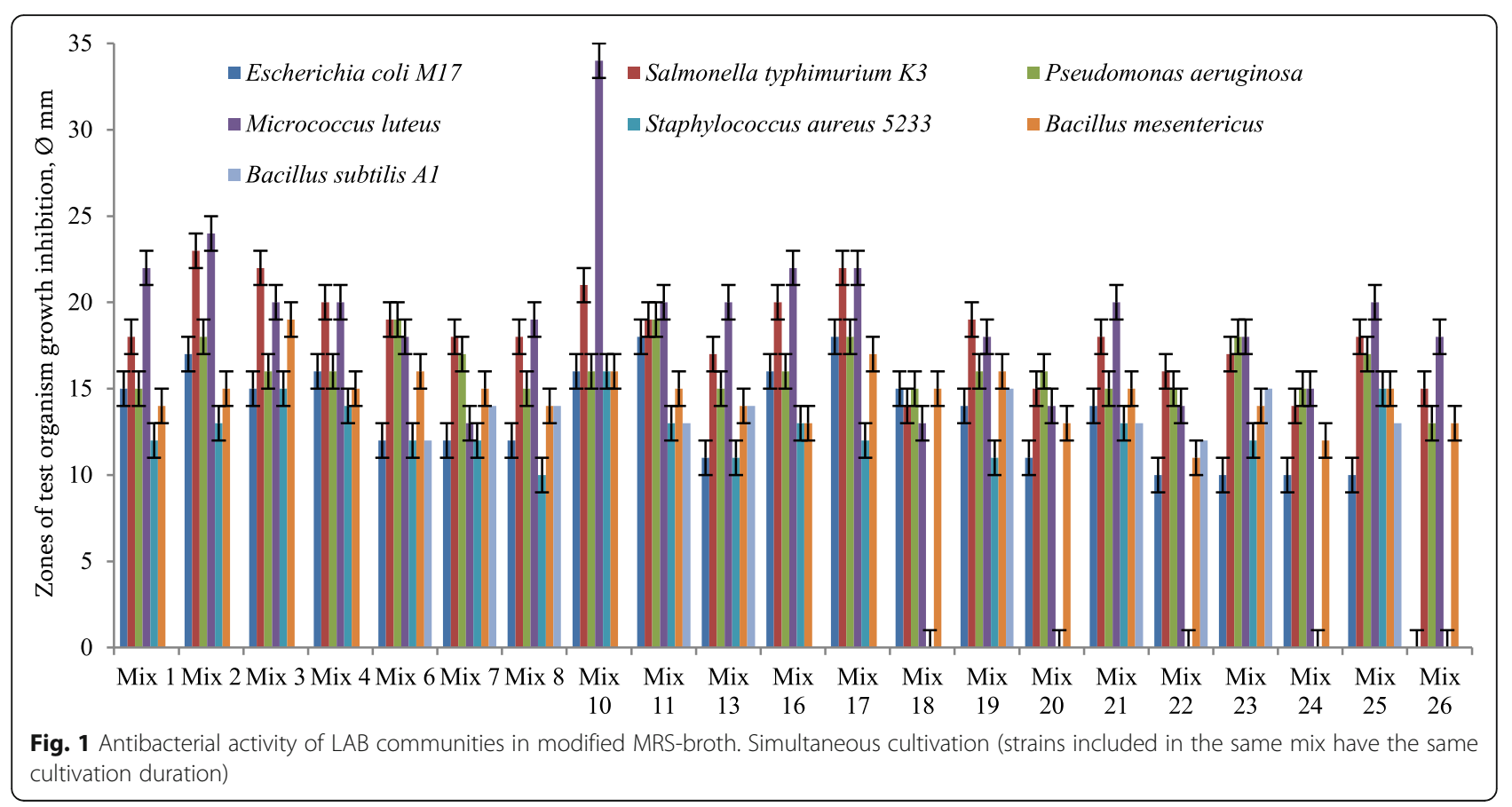

suppressed the growth of pathogens during preparation of borde. Borde is a traditional Ethiopian fermented low-alcohol beverage [7]. During the borde fermentation a lot of outside microorganisms can contaminate the product. The authors showed the presence of antibacterial activity of borde LAB strains against all these bacteria. It should be noted that the amount of test microorganisms was increased by $3 \log$ during $8 \mathrm{~h}$ cultivation in in supernatants that did not contain LAB cells. Interestingly, during simultaneous $\mathrm{LAB}$ cultivation and test organisms, the colony forming units (CFU) of LAB was increased by the same amount under these conditions.

Other authors investigated the antibacterial activity of LAB mixtures which had been composed of six different strains. All these strains were isolated from the Ethiopian fermented milk ergo [10]. They showed that the growth of $P$. aeruginosa, $S$. aureus and $S h$. boyidii was completely inhibited during 5,6 , and 7 days, respectively, after addition of the newly created $\mathrm{LAB}$ mixture into ayib.

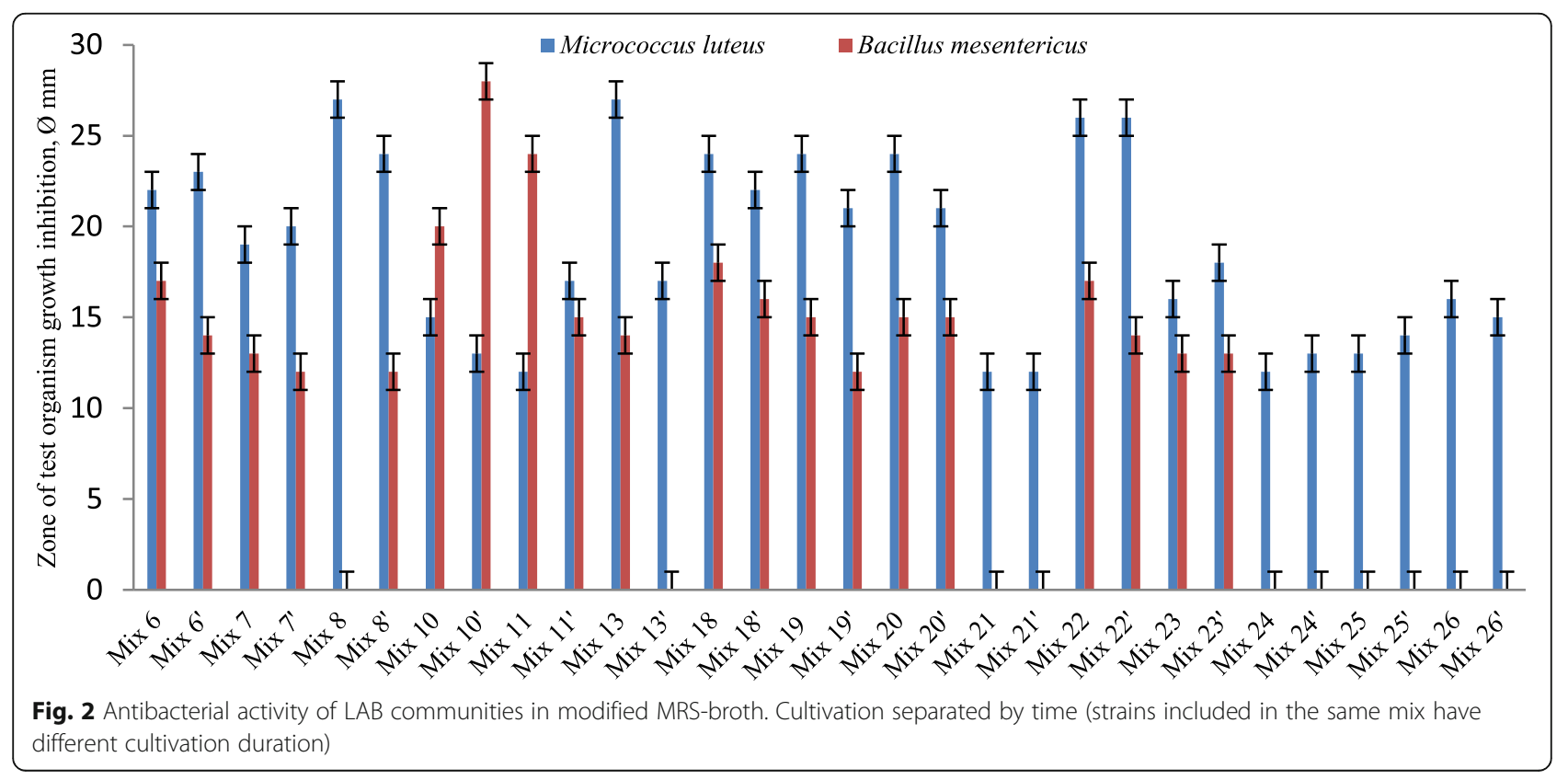




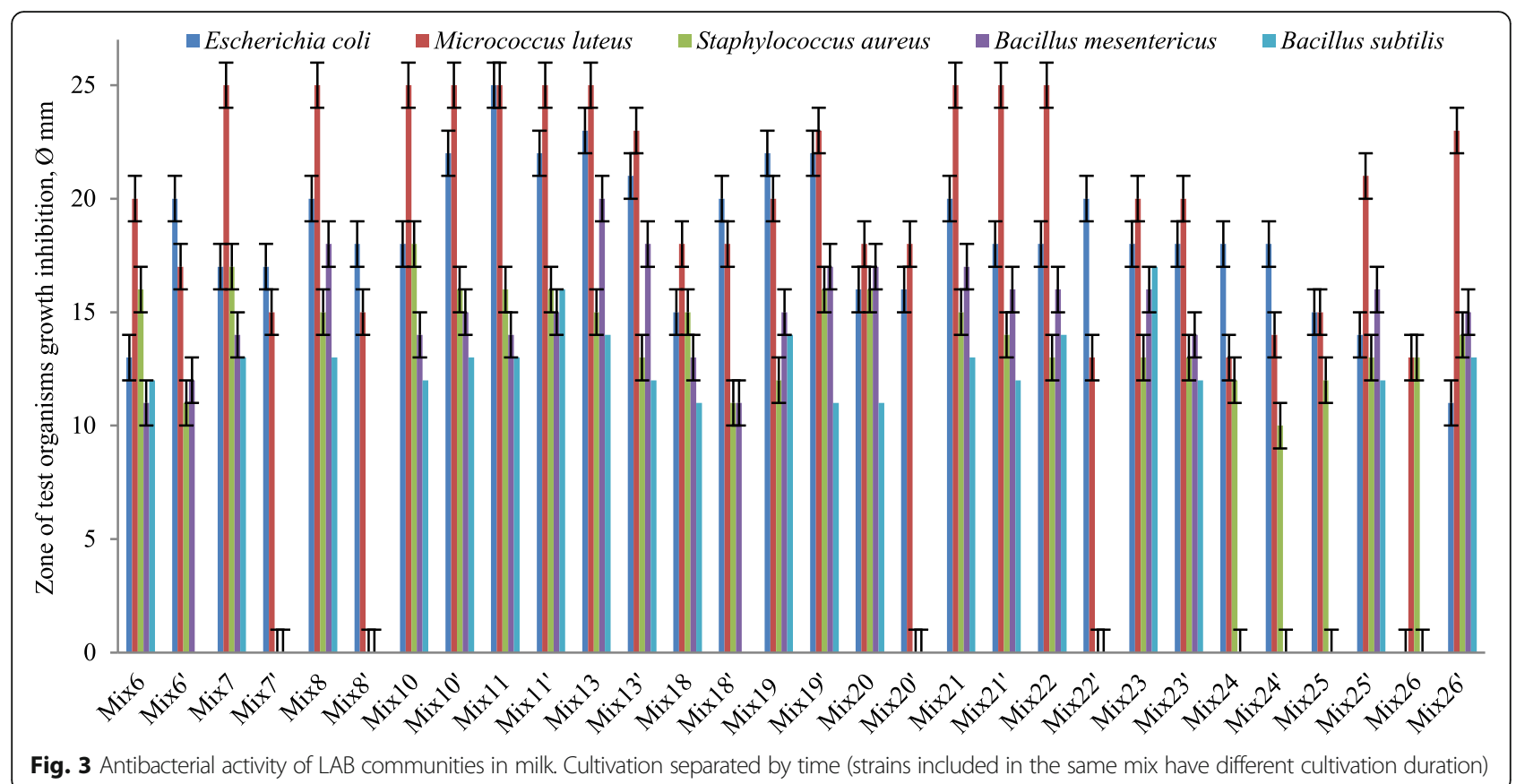

Fig. 3 Antibacterial activity of LAB communities in milk. Cultivation separated by time (strains included in the same mix have different cultivation duration)

In another work LAB strains were cultivated separately, but their cell-free cultural liquid was used for investigation of antibacterial activity [11]. The highest antimicrobial activity of cell suspension was determined in MRS after $18 \mathrm{~h}$ incubation. The results indicated that $S$. thermophilus with L. bulgaricus had the highest antibacterial effect against $S$. aureus with a zone inhibition of $10.5 \pm 0.35 \mathrm{~mm}$ and for $E$. coli with $4 \pm 0 \mathrm{~mm}$. On the other hand, our results showed the overproduction of inhibitory agents of LAB associations in the case of simultaneous cultivation in MRS. Simultaneously the antibacterial activity of various mixtures consisted of $L$. rhamnosus MDC 9661 and other LAB strains was increased from $10 \pm 2 \mathrm{~mm}$ of growth inhibition zone up to $16 \pm 1 \mathrm{~mm}$ in average against $P$. aeruginosa WT272786. Otherwise the overproduction of antibacterial components observed at the time spaced system during cultivation in milk. This may be due to production of some components, which are synthesized during the primary metabolism and used by the other bacteria, as a stimulator of antibacterial activity. This process occurs only in the presence of dairy components.

Sometimes LAB synergism can reduce their antimicrobial effect. Specifically, Domínguez-Manzano and Jiménez-Díaz [8] showed that bacteriocin production is significantly reduced in $\mathrm{LAB}$ co-cultures. Bacteriocins can be a good source of amino acids, and some LAB strains use these antibacterial components, as nutrients. This is possible due to the high proteolytic activity of strains. This mechanism allows them to survive at the presence of bacteriocin and can lead to inhibition of antagonistic activity [8]. At the same time, the activation of growth of both symbiotic strains can be observed. Similarly, the same results were obtained during our experiments, when the antifungal activity of MDC 9661 combinations with other LAB was partially decreased.

Interesting results were obtained by other researchers in the case of antifungal activity of LAB associations as well as associations of $\mathrm{LAB}$ and lactose fermenting yeasts [9]. Specifically, they showed that these associations have antagonistic activity against Penicillium notatum, Penicillium spp. and Cephalosporium humicola [9].

For centuries, Armenians have been preparing national food which has a stimulating effect on human and animal immune system, health, etc. [13]. Traditional Armenian food is rich in proteins and essential nutrients. For the production of such kind of food the rural population separated and cultivated unique LAB associations for centuries. The selection was made on the basis of their antimicrobial, proteolytic and antifungal activities. The antibacterial and proteolytic activity of some isolated strains had been shown before [14]. The antifungal effects of many LAB strains were investigated against different kinds of moulds in the previous study [12].

The antimicrobial components synthesized by LAB are in demand in industry as safe and specific bio-preservatives [15]. Many bacteriocins produced by this group of bacteria have successfully proved to be preservatives for meat products, sea food, dairy and cereal products, fermented vegetables and fruits [16]. The main approaches to use of bacteriocins, as well as intact LAB strains with high inhibitory activity are extending the shelf life of foods, immobilizing them in packing materials and adding them to other preservatives. For the confirmation of the bacteriocineous 
nature of investigated $\mathrm{LAB}$ antibacterial components, the multiple following experiments should be done. Because we used previously investigated LAB strains in all mixtures, we can only assume that in the combinations they synthesized some antibacterial components with proteinaceous nature $[14,17]$.

\section{Conclusions}

Thus, it can be concluded that LAB associations revealed a stronger inhibitory effect in milk in the case of time-spaced cultivation. Co-cultivation of some strains, such as L. rhamnosus MDC 9661, could lead to the inhibition of its antagonistic activity. Of course, for the confirmation of this and understanding of the mechanisms of counteraction between LAB strains in associations, continual and more detailed research is required.

\section{Methods}

\section{Objects of investigation}

The different $\mathrm{LAB}$ strains isolated from traditional Armenian dairy product matsoun, cheeses and honeybees' gastrointestinal tract were used as the objects of this investigation: Lactobacillus rhamnosus R-2002 (the accession number is KY054594 and submitted in GenBank) deposited at Microbial Depository Center (MDC) (WDM803) ('Armbiotechnology' Scientific and Production Center, National Academy of Sciences of Armenia, Yerevan, Armenia) under the number MDC 9661, Lactobacillus delbrueckii subsp. bulgaricus (RIN-2003-Ls), L. delbrueckii subsp. lactis INRA-2010-4.2 and L. delbrueckii subsp. bulgaricus INRA-2010-5.2 under the code numbers MDC 9632 and MDC 9633, respectively [18], Enterococcus faecium INR-2010-Tsov-G-St, Streptococcus thermophilus VKPM B-3809, Enterococcus durans (E.d - provided by Institut Nationale de la Recherche Agronomique, Nantes, France, INRA), Lactobacillus spp. (B7 - isolated from honeybees).

\section{Creation of LAB associations}

All associations were created in accordance with the method explained by Matevosyan et al. [20]. The LAB strains were cultivated in modified MRS broth $\left(10 \mathrm{gl}^{-1}\right.$ meat extract, $10 \mathrm{~g} \mathrm{~L}^{-1}$ poly-peptone, $5 \mathrm{~g} \mathrm{~L}^{-1}$ yeast extract, $20 \mathrm{~g} \mathrm{~L}^{-1}$ glucose, $2 \mathrm{gL}^{-1}$ ammonium citrate, $0.2 \mathrm{~g} \mathrm{~L}^{-1}$ $\mathrm{MgSO}_{4}, 0.05 \mathrm{~g} \mathrm{~L}^{-1} \mathrm{MnSO}_{4}, 1 \mathrm{gL}^{-1}$ Tween 80, 0.8\% agar, sterilization at $1 \mathrm{~atm} ., 15 \mathrm{~min}$ ) at $37^{\circ} \mathrm{C}$ during $24 \mathrm{~h}$. For the same purpose, the $10 \%$ milk was used (the milk powder was produced by "Katnarat" LLC, Armenia) [21].

\section{Determination of antifungal activity}

To determine the antifungal activity, the six most active LAB strains were selected, and from these strains 15 different combinations were created according to Bazukyan et al. [19] and Matevosyan et al. [20]. The antifungal activity of $E$. durans was not studied, since it is the reference strain from INRA (Nantes, France), the antifungal activity of which was shown by Ahmadova et al. [22]. Strain B7 was not included in creation of associations because it was not active. The antifungal properties of LAB associations were determined by both well-diffusion method and total diffusion into agar. It should be mentioned, that all experiments were carried out using mod MRS media since it was suitable for both the growth of fungi (molds) and the growth of LAB. Various species of molds and yeasts were used as test-organisms: Mucor plumbeus, Geotrichum candidum, Fusarium oxysporum, Cladosporium herbarum (isolated from spoiled food and provided by Biopolymers interaction assemble, function and interaction of proteins laboratory (FIPL), INRA) [22], Aspergillus flavus, Penicillium aurantioviolaceum, Penicillium spp. and Trichoderma viride (isolated from spoiled food and provided by Dr. K. Grigoryan, Yerevan State University, Yerevan, Armenia), Candida albicans 301 (isolated from clinical material), Debaryomyces hansenii. The well-diffusion method was carried out according to the method provided in [23] with using as the cultivation media Sabouraud with $0.9 \% \mathrm{w} \mathrm{v}^{-1}$ of agar. $50 \mu \mathrm{l}$ of each LAB cultural liquid (LAB had been grown in modified MRS broth at $37^{\circ} \mathrm{C}$ during $24 \mathrm{~h}$ ) was added to wells (2 different LAB strains were mixed at equal proportions). The presence of antifungal activity was determined by the absence of fungal/yeasts growth around of well (qualitative test). Using diffusion into agar, $250 \mu \mathrm{L}$ of each overnight LAB (2 different LAB strains were mixed at equal proportions) cultural liquid was added to small $(7 \mathrm{~mL})$ Petri dishes and covered by modified MRS agar. The suspension of fungal/molds spores (the amount was $10^{4}$ per $\mathrm{ml}$ ) was dropwise applied on the surface of media after $48 \mathrm{~h} \mathrm{LAB}$ cultivation. The suspensions of fungal spores were prepared according to Bazukyan et al. [19]. The antifungal activity was detected in the absence of fungal/molds growth on the surface of the medium (quantitative test). The antibacterial and antifungal activities of LAB mixtures were compared with the results of the initial strains.

\section{Determination of antibacterial activity}

Seven LAB strains were selected according to high antibacterial activity, which was studied previously $[14,18]$. The strain E. faecium INR-2010-Tsov-G-St did not show any antibacterial activity, that is why it was not included in the combinations.

The antibacterial activity of 21 different LAB associations was determined. Antibacterial properties of LAB mixed cultures were studied both in modified MRS-broth and milk by two methods of cultivation (simultaneous cultivation of LAB strains and cultivation of these strains separated by time at $37^{\circ} \mathrm{C}$ ) by agar well diffusion method [20, 23]. 
Different Gram-positive and Gram-negative representatives of various genera were used for detection of antibacterial activity: Staphylococcus aureus MDC 5233 (MDC, Armenia), Bacillus mesentericus WT, B. subtilis WT-A1 (isolated from a soil sample) and Micrococcus luteus WT (isolated from an air sample), Escherichia coli VKPM-M17 (Russian National Collection of Industrial Microorganisms, Institute of Genetics and Selection of Industrial Microorganisms, Moscow, Russia), Salmonella typhimurium MDC 1759 and Pseudomonas aeruginosa WT272786 (isolated from clinical material and provided by "Prom-Test" LLC, Yerevan, Armenia). $100 \mu \mathrm{L}$ of the mixtures was added to well. Diffusion of antibacterial substances happened during $30 \mathrm{~min}$ at room temperature. The diameters of test-organisms growth inhibition zones were measured after $24 \mathrm{~h}$. As a positive result, at least $2 \mathrm{~mm}$ in diameter, a net zone of inhibition was detected, as a positive result.

Simultaneous cultivation was carried out by mixing of each 2 separate $\mathrm{LAB}$ strains $(0.5 \%)$ in the same growth medium (modified MRS-broth) and cultivating together at $37^{\circ} \mathrm{C}$ during $24 \mathrm{~h}$. The CFU of each initial LAB strain was adjusted to $10^{8}$ before mixing. The agar well-diffusion method, which described above, has also been used in these experiments. The second way was time-spaced LAB strains cultivation (strains have different cultivation duration). At the first time, the first LAB strain was cultivated in mod MRS (or milk) during $24 \mathrm{~h}$ at $37^{\circ} \mathrm{C}$ and then mixed with the second strain and cultivated again under the same conditions. So, the first LAB strain was cultivated during $48 \mathrm{~h}$, and the second strain was cultivated during $24 \mathrm{~h}$. Then the places of strains were changed. In each case the antibacterial activity of associations was compared with the same activity of LAB pure cultures.

\section{Data processing}

All data were averages of three independent experiments. The standard errors were determined using Software Excel 2013.

\section{Abbreviations}

BIA FIPL: Biopolymers Interaction Assemble, Function and Interaction of Proteins LaboratoryINRANational Research Institute of Agronomy; LAB: lactic acid bacteria; MDC: Microbial Depository Center; MRS: DeMan, Rogosa and Sharpe; VKPM: Russian National Collection of Industrial Microorganisms, Institute of Genetics and Selection of Industrial Microorganisms

\section{Acknowledgements}

The authors thank Drs. Jean-Marc Chobert and Thomas Haertle from INRA, Nantes (France) and Dr. Karina Grigoryan from Yerevan State University, Yerevan (Armenia) for providing fungi strains.

\section{Funding}

The work was done within the framework of Basic support from Committee of Science, Ministry of Education and Science of Armenia which financial support was used for the design of the study, and supported by Research grants from the Armenian National Science and Education Fund (ANSEF) based in New York, USA, to IB (\#Biotech-3474; 4431), which financial support was used to collect samples, to analyze data and to purchase all consumables and materials.

\section{Availability of data and materials}

All data generated and/or analyzed during the current study are included in this published article. The datasets used and/or analyzed during this study are also available from the corresponding author on reasonable request.

\section{Authors' contributions}

IB was conceived and designed the experiments, performed the experiments, analyzed all data and interpreted the results, prepared and wrote the manuscript. LM performed experiments and wrote the manuscript. AT critically interpreted the results, provided discussion, revised and edited the manuscript. All authors have read and approved the manuscript.

\section{Ethics approval and consent to participate}

Not applicable.

\section{Consent for publication}

Not applicable.

\section{Competing interests}

The authors declare that there is no conflict of interest among them.

\section{Publisher's Note}

Springer Nature remains neutral with regard to jurisdictional claims in published maps and institutional affiliations.

Received: 25 February 2019 Accepted: 7 May 2019

Published online: 17 May 2019

\section{References}

1. De Muynck C, Leroya AlJ, De Maeseneirea S, Arnautb F, Soetaerta W, Vandamme EJ. Potential of selected lactic acid bacteria to produce food compatible antifungal metabolites. Microbiol Res. 2004;159:339-46.

2. Ko SH. AhnC.Bacteriocin production by LactococcuslactisKC 2386 isolated from white kimchi. Food Sci. Biotech. 2000:9(4):263-9.

3. Nes IF, Kjos M, Diep DB. Antimicrobial components of lactic acid bacteria. In: Lahtinen S, Ouwehand AC, Salminen S, von WrightA, editors. Lactic acid Bacteria - microbiological and functional aspects: $4^{\text {th }}$ edition. CRC Press,2011Chapter. 15:286-322

4. Ammor MS, Mayo B. Selection criteria for lactic acid bacteria to be used as functional starter cultures in dry sausage production: an update. Meat Sci. 2007:76:138-46.

5. Magnusson J. Antifungal activity of lactic acid bacteria. PhD thesis, Agraria 397. Swedish University of Agricultural Sciences, Uppsala, Sweden,2003.

6. Marco M. Lactobacillus plantarum in foods. In: Hirneisen KA, Kniel KE, editors. Encyclopedia of biotechnology in agriculture and food. New York: Taylor and Francis; 2011. p. 360-2.

7. Tadesse G, Ashenafi M, Ephraim E. Survival of E. coli O157:H7, Staphylococcus aureus, Shigella flexneri and Salmonella spp. in fermenting 'Borde', a traditional Ethiopian beverage. Food Cont. 2005;16:189-96.

8. Domínguez-Manzano J, Jiménez-Díaz R. Suppression of bacteriocin production in mixed species cultures of lactic acid bacteria. Food Control. 2013:30:474-9.

9. Saubenova MG, Kuznetsova TV, Khalymbetova AE, Shormanova MM. Antifungal activity of association of lactic acid bacteria: Institute of Microbiology and Virology, CS MES RK, Almaty, Republic of Kazakhstan; 2014

10. Jermen MN, Fassil AT, Antenehe TT. Evaluation of the antagonistic effect of six mixed cultures of lactic acid bacteria, isolated from the Ethiopian fermented milk ergo, against some food borne pathogens inoculated into the Ethiopian cottage cheese ayib. African J Microb Res. 2015;9(29):1789-97.

11. Ravindran L, Manjunath N, Darshan PR, Suba GA. In vitro study analysis of antimicrobial properties of lactic acid bacteria against pathogens. J Bio Innovation. 2016:5:262-9.

12. Bazukyan I, Matevosyan L. Antifungal activity of lactic acid bacteria isolated from Armenian dairy products. The FEBS J. 2014:281 Suppl., 651.

13. Hakobyan AS, Panosyan OA, Bazukyan I. Isolation and study of the properties of lactic acid bacteria from starter cultures of matsun from different regions of Armenia. Sci Notes YSU NaturSci. 2006;3:149-52.

14. Movsesyan I, Ahabekyan N, Bazukyan I, Madoyan R, Dalgalarrondo M, Chobert J, Popov Y, Haertle T. Properties and survival under simulated 
gastrointestinal conditions of lactic acid bacteria isolated from Armenian cheeses and matsouns. Biotech Biotech Equip. 2010;24:444-9.

15. Stoyanova LG, Ustyugova EA, Netrusov Al. Antibacterial metabolites of lactic acid bacteria: their diversity and properties. Appl Biochem Microbiol. 2012; 48:229-43.

16. El-GhaishSh AA, Hadji-Sfaxi I, El Mecherfi K, Bazukyan I, Choiset Y, Rabesona H, Sitohy M, Popov YG, Kuliev AA, Mozzi F. ChobertJ,HaertléT.Potential use of lactic acid bacteria for reduction of allergenicity and for longer conservation of fermented foods. Trends Food Sci Tech. 2011;20:1-8.

17. Bazukyan IL, Babayan AM, Trchounian A. Some properties of antibacterial component of lactic acid bacteria isolated from Armenian dairy products. NAS RA Electronic J of Natural Sci. 2013;2(21):39-43.

18. Keryan A, Bazukyan I, Trchounian A. Lactobacilli isolated from the Armenian fermented milk product matsoun: growth properties, antibacterial and proteolytic activity and their dependence on pH. Int J Dairy Tech. 2016;69: $1-10$.

19. Bazukyan I, Matevosyan L, Toplaghaltsyan A, Trchounian A. Antifungal activity of lactobacilli isolated from Armenian dairy products: an efective strain and its probable nature. AMB Expr. 2018;8(87):1-8.

20. Matevosyan L, Bazukyan I, Trchounian A. Comparative analysis of the effect of ca and mg ions on antibacterial activity of lactic acid bacteria isolates and their associations depending on cultivation conditions. AMB Expr. 2019; 9(32):1-11.

21. Goers L, Freemont P, Karen M, Polizzi KM. Co-culture systems and technologies: taking synthetic biology to the next level. J Royal Soc Interface. 2014:(96):20140065

22. Ahmadova A, DimitrovTodorov $\mathrm{S}$, Hadji-Sfaxi I, Choiset $Y$, Rabesona $H$, Messaoudi S, Kuliyev A, Gombossy de Melo Franco BD, Chobert J, Haertlé T. Antimicrobial and antifungal activities of Lactobacillus curvatus strain isolated from homemade Azerbaijani cheese. Anaerobe. 2013;20:42-9.

23. Ndagano D, Lamoureux T, Dortu C, Vandermoten S, Thonart P. Antifungal activity of 2 lactic acid bacteria of the Weissella genus isolated from food. J Food Sci. 2011:76:305-11.

Ready to submit your research? Choose BMC and benefit from:

- fast, convenient online submission

- thorough peer review by experienced researchers in your field

- rapid publication on acceptance

- support for research data, including large and complex data types

- gold Open Access which fosters wider collaboration and increased citations

- maximum visibility for your research: over $100 \mathrm{M}$ website views per year

At $\mathrm{BMC}$, research is always in progress.

Learn more biomedcentral.com/submissions 